\title{
Antibiotic resistance of Enterococcus species: 3-year data
}

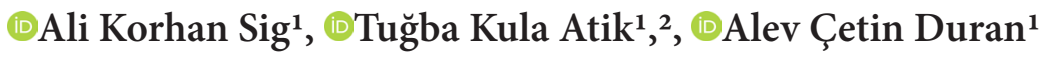 \\ ${ }^{1}$ Atatürk State Hospital, Department of Medical Microbiology, Balıkesir, Turkey \\ ${ }^{2}$ Balıkesir University, Faculty of Medicine, Department of Medical Microbiology, Balıkesir, Turkey
}

Cite this article as: Sig AK, Kula Atik T, Çetin Duran A. Antibiotic resistance of Enterococcus species: 3-year data. J Health Sci Med 2021; 4(5): 670-674.

\begin{abstract}
Aim: The aim of this study was to investigate the prevalence of Enterococcus species and to evaluate susceptibilities to antimicrobial agents in a state (secondary) hospital.

Material and Method: A total of 1676 enterococci strains (490 E. faecium, 1146 E. faecalis, 10 E. casseliflavus/E. gallinarum and 30 other Enterococcus species) isolated from cultures obtained from January 2017 to December 2019 in Balıkesir Atatürk State Hospital were included. Blood cultures were incubated in automated device (Render Biotech Co.Ltd., PRC). Other cultures were incubated with conventional methods. Grown colonies were identified by PhoenixTM 100 automated system (Becton Dickinson, USA). Identifications that need confirmation or strains identified to genus level were further evaluated with conventional techniques. Antimicrobial susceptibility tests were performed by same system, Kirby-Bauer disc diffusion and gradient strip method according to EUCAST guidelines.

Results: $43.1 \%, 27.1 \%, 14.7 \%$ and $15.1 \%$ of enterococci were isolated from urine, blood/sterile body fluids, wound/abscess and other samples. Majority of the strains were ciprofloxacin (72.0\%) and levofloxacin (74.1\%) resistant, and more than $40 \%$ showed ampicillin and high-level gentamicin resistance. Glycopeptide resistance was relatively high (5.4\%), especially when considering E. faecium (12.1\%). There was not any tigecycline and linezolid resistance.

Conclusion: Antimicrobial resistance is a serious and growing public health problem affecting all countries, which is not just a topic of medicine, but multiple sectors such as commercial companies, food industry, veterinarians, etc. High percentages of resistance strongly indicate to get a local action, which should be followed by national and global one.
\end{abstract}

Keywords: Enterococci, vancomycin, antimicrobial stewardship, EUCAST, CLSI

\section{INTRODUCTION}

Enterococci exist in most foods such as raw meat, cheese, milk, and vegetables, since they also play role in fermentation process. Due to their natural habitat, human-enterococci interaction is very tight, and they are significant members of human intestinal microbiota; however they are known to cause both healthcareassociated and community-associated infections in mild to severe spectrum, including urinary tract (UTIs) and bloodstream infections (BSIs) $(1,2)$. Enterococcus faecalis and Enterococcus faecium are the most common causative agents (>90\%) (1).

In recent years, these species also took their place in the antibiotic resistance. The Centers for Disease Control and Prevention (CDC) has declared vancomycinresistant enterococci (VRE) as a serious threat in 2019, since approximately $30 \%$ of all healthcare-associated enterococcal infections show resistance to vancomycin. Although VRE rates are high in healthcare-associated infections, community-associated infections also show such resistance, but in lower rates (3). Especially E. faecium has several mechanisms of intrinsic resistance and may show different acquired resistance mechanisms provided by gene mutations or incorporations by plasmids, transpozons, or integrons (4). In addition, despite their infrequent isolation from infection sites, some particular species such as E. gallinarum and E. casseliflavus show intrinsic resistance to several antibiotics (5).

Investigation of prevalence on isolated microorganisms from various cultures and antibiotic susceptibilities may give the physicians information about empiric therapies and may provide capability to observe significant changes during the following years. The aim of this study was to investigate the prevalence of Enterococcus species and to evaluate susceptibilities to antimicrobial agents in a state (secondary) hospital. 


\section{MATERIAL AND METHOD}

Approved by Ethics Committee of Balıkesir University, Faculty of Medicine (Date: 10.06.2020, Decision No: 2020/98). All procedures were carried out in accordance with the ethical rules and the principles of the Declaration of Helsinki.

Cultures obtained from January 2017 to December 2019 in Balıkesir Atatürk State Hospital were included in the study. The three-year period data were evaluated for isolated enterococci and their antimicrobial susceptibilities, retrospectively. A total of 1676 enterococci isolates (490 E. faecium, 1146 E. faecalis, 10 E. casseliflavus/E. gallinarum and 30 other Enterococcus species) were included in the study.

The only first sample was included for repetitious samples from the same patient and BSI episodes were determined according to CDC criteria and clinical evaluations (6). BacT/Alert ${ }^{\circledast}$ DD (bioMérieux, Marcyl'Etoile, France) and Render BC128 (Shandong Huifa Electronics Technology Co., Ltd., PRC) automated blood culture systems were used for blood cultures (BCs) and incubation period was determined according to manufacturer's recommendations. Other cultures were applied and incubated with conventional methods. Grown colonies were identified by PhoenixTM 100 automated system (Becton Dickinson, USA). Identifications that need confirmation or strains identified to genus level were further evaluated with conventional techniques. Contaminations of $\mathrm{BCs}$ were determined according to CDC criteria and clinical evaluations (6).

Antimicrobial susceptibility tests were performed by PhoenixTM 100 automated system (Becton Dickinson, USA) and by Kirby-Bauer disc diffusion and gradient strip method according to guidelines of The European Committee on Antimicrobial Susceptibility Testing (EUCAST) (7). Observed vancomycin and teicoplanin resistance and uncertain results (fuzzy zone edges) in disc diffusion were further confirmed with automated system and gradient strip method. Isolates with highlevel gentamicin resistance were further tested for highlevel streptomycin resistance.

\section{RESULTS}

A total of 1676 enterococci isolates were included in the study, and over than $97 \%$ of them were the most common causative species; $E$. faecalis ( $\mathrm{n}=1146,68.4 \%)$ and E. faecium (n=490, 29.2\%). Enterococcus-isolated cultures were predominantly from urinary tract samples ( $\mathrm{n}=722,43.1 \%)$, followed by sterile body fluids, including blood ( $\mathrm{n}=455,27.1 \%)$. Since our facility is continuously screened for VRE in specific patients, especially in the ICUs, 72 samples were rectal swabs and 39 isolates (34 E. faecium, 5 E. faecalis) were detected as vancomycin resistant, which covers approximately a half of such resistant strains. The majority of other VRE were isolated from urinary tract $(\mathrm{n}=19)$ and blood $(\mathrm{n}=12)$ samples. Despite relatively high resistance rates to quinolones and aminoglycosides, there was no linezolid and tigecycline resistant strain.

All results were presented in Table $\mathbf{1}$ and 2, comparison with The Turkish National Antimicrobial Resistance Surveillance System (UAMDSS) was presented in Table 3.

\section{DISCUSSION}

Enterococcus, with over than 50 species, are natural inhabitants of humans and animals; however, they remain to be important pathogens of human infections. UTIs, intraabdominal abscesses and BSIs are major manifestations, in addition these species may cause healthcare-associated infections, including biofilm formations on medical devices $(8,9)$.

Enterococci also hold major importance due to several intrinsic resistance and ability to create acquired resistance rapidly. Glycopeptide resistance is the major problem that particularly E. faecium strains show this resistance. Since VRE is a public health issue, these strains should be continuously under surveillance of infection control committees (10). Recently, several types of glycopeptide resistance were defined (VanA, B, C, D, E, F, G, L, M, N). Van A and B are the most frequent ones; however, their level of resistance varies (4). VRE strains also show higher resistance rates to other antibiotics, such as gentamicin and

\begin{tabular}{|c|c|c|c|c|c|c|}
\hline Sample / Species & $\begin{array}{c}\text { E. faecium } \\
(\mathrm{n}=490,29.2 \%)\end{array}$ & $\begin{array}{c}\text { E. faecalis } \\
(\mathrm{n}=1146,68.4 \%)\end{array}$ & $\begin{array}{l}\text { E. casseliflavus/ } \\
\text { E. gallinarum } \\
(\mathrm{n}=10,0.6 \%)\end{array}$ & $\begin{array}{c}\text { Other } \\
\text { Enterococcus spp. } \\
(\mathrm{n}=30,1.8 \%)\end{array}$ & \multicolumn{2}{|c|}{ Overall $(\mathbf{n}, \%)$} \\
\hline Blood and Other Sterile Body Fluids & 146 & 292 & 4 & 12 & 454 & 27.1 \\
\hline Urine & 190 & 517 & 3 & 11 & 721 & 43.0 \\
\hline Wound/Abscess & 57 & 182 & 0 & 7 & 246 & 14.7 \\
\hline Respiratory (Upper, Lower) Samples & 10 & 13 & 0 & 0 & 23 & 1.4 \\
\hline Other (e.g., rectal swab) & 87 & 142 & 3 & 0 & 232 & 13.7 \\
\hline Total & 490 & 1146 & 10 & 30 & 1676 & 100 \\
\hline
\end{tabular}




\begin{tabular}{|c|c|c|c|c|c|c|c|c|c|c|c|c|c|c|c|}
\hline \multirow[t]{2}{*}{ Antibiotics / Species } & \multicolumn{3}{|c|}{$\begin{array}{c}\text { E. faecium } \\
(\mathrm{n}=490,29.2 \%)\end{array}$} & \multicolumn{3}{|c|}{$\begin{array}{c}\text { E. faecalis } \\
(\mathrm{n}=1146,68.4 \%)\end{array}$} & \multicolumn{3}{|c|}{$\begin{array}{c}\text { E. casseliflavus/ } \\
\text { E. gallinarum } \\
(\mathrm{n}=10,0.6 \%)\end{array}$} & \multicolumn{3}{|c|}{$\begin{array}{c}\text { Other } \\
\text { Enterococcus spp. } \\
(\mathrm{n}=30,1.8 \%)\end{array}$} & \multicolumn{3}{|c|}{ Overall } \\
\hline & $\begin{array}{c}\mathrm{S} \\
(\mathbf{n})\end{array}$ & $\begin{array}{c}\mathbf{R} \\
(\mathbf{n})\end{array}$ & $\begin{array}{c}\text { R-Rate } \\
(\%)\end{array}$ & $\begin{array}{c}\mathrm{S} \\
(\mathbf{n})\end{array}$ & $\begin{array}{c}\mathbf{R} \\
(\mathbf{n})\end{array}$ & $\begin{array}{c}\text { R-Rate } \\
(\%)\end{array}$ & $\begin{array}{c}\mathrm{S} \\
(\mathbf{n})\end{array}$ & $\begin{array}{c}\mathbf{R} \\
(\mathbf{n}) \\
\end{array}$ & $\begin{array}{c}\text { R-Rate } \\
(\%)\end{array}$ & $\begin{array}{c}S \\
(n)\end{array}$ & $\begin{array}{c}\mathbf{R} \\
(\mathbf{n})\end{array}$ & $\begin{array}{c}\text { R-Rate } \\
(\%)\end{array}$ & $\begin{array}{l}\mathrm{S} \\
(\mathbf{n})\end{array}$ & $\begin{array}{c}\mathbf{R} \\
(\mathbf{n})\end{array}$ & $\begin{array}{c}\text { R-Rate } \\
(\%)\end{array}$ \\
\hline Ampicillin ${ }^{b}$ & 64 & 379 & 85.6 & 921 & $23^{c}$ & 2.4 & 8 & 2 & 20.0 & 19 & 7 & 26.9 & 1012 & 411 & 28.9 \\
\hline Levofloxacin $^{\mathrm{a}}$ & 30 & 155 & 83.8 & 145 & 353 & 70.9 & 2 & 0 & None & 1 & 0 & None & 178 & 508 & 74.1 \\
\hline Ciprofloxacin ${ }^{a}$ & 31 & 159 & 83.7 & 160 & 351 & 68.7 & 2 & 0 & None & 6 & 1 & 14.3 & 199 & 511 & 72.0 \\
\hline Teicoplanin & 390 & 54 & $12.1^{\mathrm{c}}$ & 927 & 23 & $2.4^{\mathrm{c}}$ & 10 & 0 & None & 24 & 1 & 4.0 & 1354 & 78 & 5.4 \\
\hline Vancomycin & 390 & 54 & $12.1^{\mathrm{c}}$ & 936 & 23 & $2.4^{\mathrm{c}}$ & & NA & & 24 & 1 & 4.0 & 1350 & 78 & 5.4 \\
\hline Linezolid & 443 & 0 & None & 941 & 0 & None & 10 & 0 & None & 24 & 0 & None & 1418 & 0 & None \\
\hline Gentamicin (high-level) $^{1}$ & 284 & 206 & 42.0 & 679 & 467 & 40.8 & 8 & 2 & 20.0 & 19 & 5 & 20.8 & 990 & 680 & 40.7 \\
\hline Streptomycin (high-level) ${ }^{1}$ & 81 & 338 & 80.7 & 508 & 360 & 41.5 & 5 & 5 & 50.0 & 21 & 9 & 30.0 & 615 & 712 & 53.7 \\
\hline Tigecycline & 95 & 0 & None & 740 & 0 & None & 5 & 0 & None & & ID & & 840 & 0 & None \\
\hline Amoxicillin \& Clavulanic acid ${ }^{b}$ & 16 & 90 & 84.9 & 289 & 12 & 3.9 & 1 & 0 & None & 11 & 1 & 8.3 & 317 & 103 & 24.5 \\
\hline Ampicillin \&Sulbactam ${ }^{\mathrm{b}}$ & 4 & 2 & 33.3 & 85 & 2 & 2.3 & 1 & 0 & None & 4 & 1 & 20.0 & 94 & 5 & 5.1 \\
\hline Nitrofurantoin ${ }^{a}$ & & NA & & 496 & 21 & 4.1 & & NA & & & NA & & & NA & \\
\hline Quinupristin-dalfopristin & 240 & 70 & 22.6 & & NA & & & NA & & & NA & & & NA & \\
\hline
\end{tabular}

\section{Table 3. Comparison of UAMDSS and present study (17)}

\begin{tabular}{|c|c|c|c|c|c|c|c|}
\hline \multirow{2}{*}{ Years } & \multirow{2}{*}{$\begin{array}{l}\text { Present } \\
\text { Studyd }\end{array}$} & \multicolumn{6}{|c|}{ UAMDSS } \\
\hline & & $2011^{c}$ & $2012^{c}$ & $2013^{c}$ & $2014^{c, d}$ & $2015^{c, d}$ & $2016^{\mathrm{cd} d}$ \\
\hline Antibiotics / E. faecium & & & & -Rate (9) & & & \\
\hline Ampicillin ${ }^{\mathrm{a}}$ & 85.6 & 88.1 & 85.3 & 100 & 82.0 & 87.0 & 91.6 \\
\hline Teicoplanin $^{\mathrm{b}}$ & 12.1 & 0.3 & 16.0 & 18.4 & ID & ID & ID \\
\hline Vancomycin ${ }^{\mathrm{b}}$ & 12.1 & 17.0 & 16.7 & 22.8 & 16.0 & 16.0 & 16.0 \\
\hline Linezolid & None & 0.6 & 2.7 & 1.1 & 4.0 & 4.0 & 1.0 \\
\hline Gentamicin (high-level) & 42.0 & 52.3 & 51.2 & 43.6 & 43.0 & 69.0 & 61.7 \\
\hline Streptomycin (high-level) & $80.7^{1}$ & 49.0 & 36.0 & 47.3 & ID & ID & ID \\
\hline Antibiotics /E. faecalis & & & & -Rate $(9$ & & & \\
\hline Ampicillin ${ }^{\mathrm{a}}$ & 2.4 & 9.7 & None & 4.7 & 8.0 & 9.0 & 6.0 \\
\hline Teicoplanin ${ }^{\mathrm{b}}$ & 2.4 & 0.3 & None & 0.2 & ID & ID & ID \\
\hline Vancomycin ${ }^{\mathrm{b}}$ & 2.4 & 0.9 & 0.6 & 0.9 & 3.0 & 3.0 & 1.3 \\
\hline Linezolid & None & 0.4 & 2.0 & 0.8 & 3.0 & 2.0 & None \\
\hline Gentamicin (high-level) & 40.8 & 29.2 & 31.8 & 21.4 & 22.0 & 54.0 & 57.2 \\
\hline Streptomycin (high-level) & $41.5^{1}$ & 31.1 & 23.3 & 26.0 & ID & ID & ID \\
\hline
\end{tabular}

streptomycin (11). Linezolid resistance is another problem, which is claimed to be related to antibiotic exposure such as in staphylococci; however, resistant strains were also detected in cases without any such kind of history (10). In addition, particular species (E. casseliflavus/E. gallinarum) shows intrinsic resistance to specific antibiotics (5). Interestingly, resistance to a particular antibiotic is not only associated with its usage individually, since cross-resistance depending on the consumption of other antibiotics was also reported (e.g.; cephalosporins and vancomycin) (12).
Several studies focused on the origin and resistance of enterococci. One of the widest studies in European countries is the "The Central Asian and European Surveillance of Antimicrobial Resistance Network (CAESAR)" report, which indicates a serious burden of enterococcal vancomycin resistance especially in the eastern area, including Turkey (VR-E. faecium 10-25\% interval); furthermore, who declared VR-E. faecium as one of the high-priority pathogens that urgently needs new antibiotics for treatment $(13,14)$. Our findings are similar to CAESAR report for both E. faecium and E. faecalis regarding ampicillin $(85.6 \%$ \& $2.4 \%$ vs. $86 \%$ \& 
$4 \%$ ), high-level gentamicin ( $42 \%$ \& $40.8 \%$ vs. $55 \%$ \& $37 \%)$, vancomycin $(12.1 \% \& 2.4 \%$ vs. $14 \% \& 1 \%)$ and linezolid (None vs. None) resistance. Our higher rates for VR-E. faecalis can be explained with the CAESAR sample spectrum, since only cerebrospinal fluid and $\mathrm{BC}$ results are included in CAESAR surveillance, but our findings also cover rectal swab screenings. In another study focusing on 10-year BC data in Turkey, while glycopeptide (from $6.2 \%$ to $15 \%$ ) and ampicillin resistance (from $36.7 \%$ to $46 \%$ ) increased, high-level gentamicin resistance dropped (from $66.4 \%$ to $39 \%$ ) for all Enterococcus spp. The authors claimed this because of changing prescribing obligations in their facility regarding gentamicin consumption (15). In a wide study in 2016, Enterococcus spp. were isolated in 2.9\% of 7-years of BCs and ampicillin resistance was 75$100 \%$ while vancomycin resistance was even $32.3 \%$ in 2010 (2008-2014; 0-32.3\%) for E. faecium (E. faecalis; ampicillin resistance $3.7-16.2 \%$, vancomycin resistance $0-5.9 \%)(16)$. In a meta-analysis from Turkey focusing data of 2000-2015; ampicillin, vancomycin, high-level gentamicin, high-level streptomycin, ciprofloxacin, levofloxacin and linezolid resistance of $E$. faecalis was found $24.7 \pm 29.4 \%, 2.2 \pm 1.0 \%, 37.1 \pm 17.1 \%, 43.2 \pm 18.3 \%$, $41.0 \pm 20.8 \%, 44.6 \pm 20.5 \%$ and $1.9 \pm 2.6 \%$, respectively, and for E. faecium, they were found as $82.5 \pm 16.6 \%$, $10.3 \pm 11.3 \%, \quad 58.7 \pm 13.4 \%, \quad 74.4 \pm 8.1 \%, \quad 77.5 \pm 17.4 \%$, $21.0 \%$ and $2.4 \%$, respectively (1). These high rates of VRE do not reflect a recent problem since Canadian surveillance of CANWARD, CDC, ECDC and WHO has continuously monitored it for several years. For that matter, VRE is among the serious threats in many national surveillance studies. Turkish public health authorities performed UAMDSS project, which showed glycopeptide, aminoglycoside and linezolid resistance of E. faecium and E. faecalis steadily increased (Table 3) (17). Even though the same sample spectrum issue is also valid for UAMDSS, our data seem compatible. Additionally, it is notable that particularly our glycopeptide and fluoroquinolone resistance showed an increasing trend in this three-year period, however statistical analysis was not performed (data not shown).

High-level gentamicin resistant strains may not indicate high-level resistance to streptomycin (5). Therefore, in our study, only strains detected as gentamicin resistant were further evaluated for streptomycin resistance. So, levels of streptomycin resistance (53.7\%) seem to be relatively higher than gentamicin resistance (40.7\%); however, this situation should be considered in this regard.

The issue in comparison between previous results (e.g., UAMDSS) and this study is the methodological difference. This study is based on EUCAST techniques, while many other studies worldwide, including Turkey, were often based on The Clinical \& Laboratory Standards InstituteCLSI. It is possible to observe lower susceptibility results with EUCAST, thus our resistance rates may seem to be slightly higher (18). However, comparison of these two methods is beyond the scope of this study, and both methods actually indicate therapeutic success, so we believe our results will be good local predictors for such information and a good source of data for national surveillance.

Antibiotic consumption has been strongly associated with resistance to such pitch that WHO has been performing surveillance (e.g., European Surveillance of Antimicrobial Consumption-ESAC Project), furthermore CLSI and Turkish Microbiology Society (TMC) endorse laboratories to limit reporting of susceptibility results according to local and/or national antimicrobial stewardship programs (19-21). In the OECD report, Turkey had the highest antibiotic consumption rates in 2015, and despite all efforts, Turkey's statistics on antibiotic consumption shows only limited success. Turkish authorities applied a national action plan regarding drug use for 2014-2017 (22). On the other hand, close monitoring of hospital antibiotic use with local antimicrobial resistance surveillance seems crucial to reflect national policies to hospital level.

\section{CONCLUSION}

Antimicrobial resistance is a serious and growing public health problem affecting all countries and multiple sectors. There is an increasing trend of awareness to this issue; however, the fight against it is a multiple-stage approach starting with determining the scope of the problem, which is crucial to monitoring and create an effective response. Standardization and continuousness of antimicrobial susceptibility testing in clinical practice, and accordingly collecting reliable data on antimicrobial resistance are the first stages, which should start from local health facilities.

The data in this study should be interpreted with caution since they may not fully represent the current national status, but they just give clues for results of a comprehensive surveillance system. However, high percentages of resistance strongly indicate the need of a local action, followed by national and global ones. We believe this data will encourage laboratories and clinicians to pay more attention in following and applying national and global antimicrobial stewardship policies.

\section{ETHICAL DECLARATIONS}

Ethics Committee Approval: Approved by Ethics Committee of Balıkesir University, Faculty of Medicine (Date: 10.06.2020, Decision No: 2020/98). 
Informed Consent: Because the study was designed retrospectively, no written informed consent form was obtained from patients.

Referee Evaluation Process: Externally peer-reviewed.

Conflict of Interest Statement: The authors have no conflicts of interest to declare.

Financial Disclosure: The authors declared that this study has received no financial support.

Author Contributions: All of the authors declare that they have all participated in the design, execution, and analysis of the paper, and that they have approved the final version.

Acknowledgements: The authors wish to declare special thanks to Onur IRMAK, M.D. (Ataturk State Hospital, Department of Medical Microbiology, Balikesir, Turkey) for his precious support.

\section{REFERENCES}

1. Kilbas I, Ciftci IH. Antimicrobial resistance of Enterococcus isolates in Turkey: A meta-analysis of current studies. J Glob Antimicrob Res 2018; 12: 26-30.

2. Calonico C, Pesavento G, Delfino V, Forni S, Nostro AL. Prevalence of Antibiotic Resistance in Enterococci: A 14 Year Survey. J Food Nutr Res 2018; 6: 626-37.

3. Centers for Disease Control and Prevention (CDC). Antibiotic Resistance Threats in the United States, 2019. Atlanta, GA: U.S Department of Health and Human Services, CDC; 2019. (doi: $10.15620 / c d c: 82532)$

4. Sieńko A, Czaban S, Ojdana D, et al. Comparison of antibiotic resistance and virulence in vancomycin-susceptible and vancomycin-resistant Enterococcus faecium strains. J Med Sci 2018; 87: 195-203.

5. The European Committee on Antimicrobial Susceptibility Testing (EUCAST). Intrinsic resistance and unusual phenotypes version 3.2. Available at https://www.eucast.org/fileadmin/src/media/ PDFs/EUCAST_files/Expert_Rules/2020/Intrinsic_Resistance and_Unusual_Phenotypes_Tables_v3.2_20200225.pdf (Date of Access: 31 May 2020).

6. Centers for Disease Control and Prevention (CDC). Bloodstream Infection Event (Central Line-Associated Bloodstream Infection and non-central line-associated Bloodstream Infection). Available at https://www.cdc.gov/nhsn/PDFs/pscManual/4PSC_ CLABScurrent.pdf (Date of Access: 2 Feb 2020).

7. The European Committee on Antimicrobial Susceptibility Testing (EUCAST). Breakpoint tables for interpretation of MICs and zone diameters version 11.0. Available at https://www.eucast.org/ clinical_breakpoints/ (Date of Access: 16 December 2020).

8. Ahmed MO, Baptiste KE. Vancomycin-resistant enterococci: a review of antimicrobial resistance mechanisms and perspectives of human and animal health. Microb Drug Resist 2018; 24: 590 606.

9. Shridhar S, Dhanashree B. Antibiotic Susceptibility Pattern and Biofilm Formation in Clinical Isolates of Enterococcus spp. Interdiscip Perspect Infect Dis 2019. doi: 10.1155/2019/7854968.

10. Miller WR, Murray BE, Rice LB, Arias CA. Vancomycin-Resistant Enterococci: Therapeutic Challenges in the $21^{\text {st }}$ Century. Infect Dis Clin North Am 2016; 30: 35-9.

11. Baldir G, Engin DO, Kucukercan M, et al. High-level resistance to aminoglycoside, vancomycin, and linezolid in Enterococci strains. J Microb Infect Dis 2013; 3: 100-3.
12. Pourmand A, Mazer-Amirshahi M, Jasani G, May L. Emerging trends in antibiotic resistance: implications for emergency medicine. Am J Emerg Med 2017; 35: 1172-6.

13. World Health Organization (WHO). Central Asian and European Surveillance of Antimicrobial Resistance (CAESAR), Annual 2019 Report, 2019. Available at https://www.euro.who.int/_data/ assets/pdf_file/0003/418863/53373-WHO-CAESAR-annualreport-2019.pdf (Date of Access: 16 December 2020).

14.https://www.who.int/news/item/27-02-2017-who-publisheslist-of-bacteria-for-which-new-antibiotics-are-urgently-needed (Date of Access: 16 December 2020).

15. Mataj V, Guney M, Sig AK, et al. An Investigation into bacterial bloodstream infections and antibiotic resistance profiles in a tertiary hospital for a ten-year period. Clin Lab 2020; 66: 1467-77.

16. Gandra S, Mojica N, Klein EY, et al. Trends in antibiotic resistance among major bacterial pathogens isolated from blood cultures tested at a large private laboratory network in India, 2008-2014. Int J Infect Dis 2016; 50: 75-82.

17.https:/hsgm.saglik.gov.tr/tr/uamdss (Date of Access: 16 December 2020).

18. Cusack TP, Ashley EA, Ling CL, et al. Impact of CLSI and EUCAST breakpoint discrepancies on reporting of antimicrobial susceptibility and AMR surveillance. Clin Microb Infect 2019; 25: 910-1.

19. Versporten A, Bolokhovets G, Ghazaryan L, et al. Antibiotic use in Eastern Europe: a cross-national database study in coordination with the WHO Regional Office for Europe. Lancet Infect Dis 2014; 14: 381-7.

20.CLSI. Performance Standards for Antimicrobial Susceptibility Testing; CLSI document M100-30th Edition. Weinstein, MP: Clinical and Laboratory Standards Institute; 2020.

21.https://www.tmc-online.org/userfiles/file/26-37.pdf (Date of Access: 16 December 2020).

22.Isler B, Keske Ş, Aksoy M, et al. Antibiotic overconsumption and resistance in Turkey. Clin Microb Infect 2019; 25: 651-3. 